\title{
Reduction of acoustic-phonon deformation potential in one-dimensional array of Si quantum dot interconnected with tunnel oxides
}

\author{
Shigeyasu Uno ${ }^{\text {a) }}$ \\ Hitachi Cambridge Laboratory, Hitachi Europe Ltd., Cavendish Laboratory, Madingley Road, Cambridge, \\ CB3 OHE, United Kingdom \\ Nobuya Mori \\ Department of Electronic Engineering, Osaka University, 2-1 Yamada-oka, Suita, Osaka 565-0871, Japan \\ Kazuo Nakazato ${ }^{\text {b) }}$ \\ Department of Electrical Engineering and Computer Science, Graduate School of Engineering, Nagoya \\ University, Furo-cho, Chikusa-ku, Nagoya 464-8603, Japan \\ Nobuyoshi Koshidab) \\ Division of Electronic and Information Engineering, Faculty of Technology, Tokyo University of Agriculture \\ and Technology, Tokyo 184, Japan \\ Hiroshi Mizuta ${ }^{\text {b) }}$ \\ Department of Physical Electronics, Tokyo Institute of Technology, 2-12-1 O-okayama, Meguro-ku, Tokyo \\ 152-8552, Japan
}

(Received 25 October 2004; accepted 24 March 2005; published online 23 May 2005)

The scattering potential for the acoustic deformation potential scattering in a one-dimensional silicon quantum dot array interconnected by thin oxide layers is theoretically investigated. One-dimensional phonon normal modes are numerically obtained using the linear atomic chain model. The strain caused by an acoustic-phonon vibration is absorbed by the oxide layers, resulting in the reduction of the strain in the Si dots. This effect eventually leads to $\sim 40 \%$ reduction of the scattering potential all over the structure. The amount of the reduction does not depend on the phonon energy, but rather on the ratio of the Si dot size to the oxide thickness. (C) 2005 American Institute of Physics. [DOI: 10.1063/1.1913799]

\section{INTRODUCTION}

Recently there appeared reports on peculiar characteristics of Si quantum dots interacting with each other through thin silicon oxide layers. A series of reports suggests that electron energy loss may be strongly suppressed in nanocrystalline silicon $(n c-\mathrm{Si})$ dot arrays embedded in a silicon oxide network. ${ }^{1-5}$ Also reported is coherent coupling of electronic quantum states in $n c$-Si dots interacting through thin silicon suboxide grain boundaries, which may be associated with quasimolecular states. ${ }^{6}$ Another report proposes a nano electromechanical memory device using $n c$-Si dots embedded in a self-supporting $\mathrm{SiO}_{2}$ beam. ${ }^{7}$ However, the electrical/ mechanical properties of such systems have yet to be well understood. In particular, the electron-phonon interaction is of great importance because it is the main concern for roomtemperature device operation.

In this work we theoretically investigate phonon waves and electron-phonon interaction in a one-dimensional silicon quantum dot array interconnected by thin oxide layers (1DSiQDA). The phonon normal modes are numerically obtained using the linear atomic chain model. Approximate expressions for the acoustic deformation potential (ADP) scat-

\footnotetext{
a) Also at CREST JST (Japan Science and Technology), Shibuya TK Bldg., 3-13-11 Shibuya, Shibuya-ku, Tokyo 150-0002, Japan; electronic mail: drsuno@phy.cam.ac.uk

b) Also at CREST JST (Japan Science and Technology), Shibuya TK Bldg., 3-13-11 Shibuya, Shibuya-ku, Tokyo 150-0002, Japan.
}

tering in the 1DSiQDA are developed. The scattering potential is then evaluated and its silicon dot size dependence is analyzed in detail.

This work is a part of an attempt to reveal electron transport in the 1DSiQDA. The main objective is to predict results in the best-case scenario by investigating the most idealized structure, that is, free of interface states, perfect periodicity, perfectly straight array, and so on. In fact, some experimental observations suggest that the good periodicity and one dimensionality of the array are essential to the highenergy electron generation in the $n c$-Si system. ${ }^{8-10}$ Therefore, our calculation would eventually give the estimate of the maximum performance which actual devices can achieve. In the near future it might be possible to fabricate the ideal 1DSiQDA by using state-of-the-art nanofabrication techniques.

This paper proceeds as follows. In Sec. II theoretical backgrounds of the phonons and the ADP scattering in the 1DSiQDA are presented. Simulation method is also shown in this section. We then present simulation results and analysis in Sec. III. After discussions on several issues in Sec. IV, we summarize our conclusions in Sec. V.

\section{THEORY AND SIMULATION}

\section{A. Model}

Figure 1 shows a schematic illustration of our idealized model of the 1DSiQDA. The $x, y$, and $z$ axes are set as shown in the figure, and the $\mathrm{Si}$ dots are modeled as cubes of 


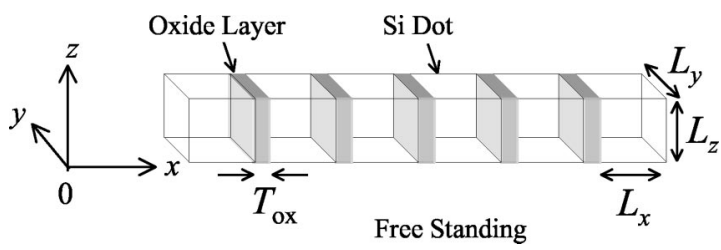

FIG. 1. Schematic illustration of one-dimensional silicon quantum dot array interconnected by thin oxide layer (1DSiQDA). The periodic structure extends infinitely in the $x$ direction.

$L_{x}, L_{y}$, and $L_{z}$ each side. These $\mathrm{Si}$ dots are interconnected by oxide layers of thickness $T_{\mathrm{ox}}$. This array of $\mathrm{Si}$ dots extends infinitely in the $x$ direction, and it is surrounded by vacuum or a material soft enough to be considered as free boundary (e.g., gas, liquid).

Table I compares the 1DSiQDA with one-dimensional GaAs quantum dot array interconnected by AlGaAs layer (1DGaAsQDA) (Ref. 11) and the silicon quantum wire (SiQW), in terms of electron-phonon interaction and acoustic-phonon wave properties. The electron-phonon interaction in the 1DGaAsQDA was theoretically investigated by Noguchi et al.," and the major phonon-scattering mechanism is polar-optical phonon (POP) scattering. On the other hand, silicon is a nonpolar material, so that the major phonon scattering mechanism in the SiQW is the acoustic deformation potential (ADP) scattering. ${ }^{12,13}$ This would also be true in the 1DSiQDA, as the oxide layer is thin compared to the Si dot size. The acoustic-phonon waves in the 1DGaAsQDA are the same as those in the GaAs quantum wire, because the mechanical properties of GaAs and AlGaAs are almost identical. However, it is not the case in the 1DSiQDA, because there is considerable difference in the mechanical properties of the Si and the oxide layer; the Young's modulus for Si and $\mathrm{SiO}_{2}$ films is 180 and $70 \mathrm{GPa}$, respectively. ${ }^{14,15}$ Thus, the 1DSiQDA is different from any other existing nanostructures, and a new theory must be developed.

\section{B. Phonon normal modes}

In general, phonon vibrations at the $\alpha_{\text {th }}$ atom in the $l_{\text {th }}$ unit cell can be written as ${ }^{16}$

$$
\mathbf{S}_{l \alpha}=\sum_{\mathbf{q}} \sqrt{\frac{\hbar}{2 \omega_{\mathbf{q}}}}\left(a_{\mathbf{q}}+a_{-\mathbf{q}}^{+}\right) \frac{1}{\sqrt{M_{\alpha}}} \mathbf{u}_{l \alpha \mathbf{q}},
$$

where $M_{\alpha}$ is the atomic mass, $\mathbf{q}$ is the phonon wave vector, $\omega_{\mathbf{q}}$ is the frequency of the phonon vibration, and $a_{-\mathbf{q}}^{+}$and $a_{\mathbf{q}}$ are the creation and annihilation operators, respectively. The vectors $\mathbf{u}_{l \alpha \mathbf{q}}$ satisfy the following orthonormalization conditions:

TABLE I. Comparison of the 1DSiQDA with one-dimensional GaAs quantum dot array interconnected by AlGaAs layer (1DGaAsQDA) and the silicon quantum wire (SiQW).

\begin{tabular}{ccc}
\hline \hline & Phonon scattering & Acoustic phonons \\
\hline 1DGaAsQDA & POP & Those in quantum wire \\
SiQW & ADP & Those in quantum wire \\
1DSiQDA & ADP & Specific to 1DSiQDA \\
\hline \hline
\end{tabular}

$$
\sum_{l \alpha} \mathbf{u}_{l \alpha \mathbf{q}}^{*} \cdot \mathbf{u}_{l \alpha \mathbf{q}^{\prime}}=\delta_{\mathbf{q q}^{\prime}}
$$

We now aim to find approximate expression for the $\mathbf{u}_{l \alpha \mathbf{q}}$ in the 1DSiQDA. According to Morse, ${ }^{17} \mathbf{u}_{l \alpha \mathbf{q}}$ in a freestanding wire with rectangular cross sections can be written as a plane wave in the $x$ direction multiplied by a function of $y$ and $z$ as

$$
\mathbf{u}_{l \alpha \mathbf{q}} \propto \mathbf{v}_{\mathbf{q}}\left(y_{l \alpha}, z_{l \alpha}\right) e^{i q_{x} x_{l \alpha}},
$$

where $\left(x_{l \alpha}, y_{l \alpha}, z_{l \alpha}\right)$ denotes the equilibrium point of the $\alpha_{\text {th }}$ atom in the $l_{\text {th }}$ unit cell. The vector $\mathbf{v}_{\mathbf{q}}\left(y_{l \alpha}, z_{l \alpha}\right)$ includes the trigonometric functions and is often approximated as plane waves having antinodes at the side edge of the structure. ${ }^{11,18,19}$ This is also true in the 1DSiQDA. However, the $x$ component is no longer a plane wave because of the large mechanical mismatch between the Si dots and the oxide layers, as discussed in the previous subsection. Therefore, the phonon wave functions in the 1DSiQDA are written as

$$
\mathbf{S}_{l \alpha}=\sum_{\mathbf{q}} \sqrt{\frac{\hbar}{2 \omega_{\mathbf{q}}}}\left(a_{\mathbf{q}}+a_{-\mathbf{q}}^{+}\right) \frac{1}{\sqrt{M_{\alpha} N_{y} N_{z}}} e^{i \mathbf{Q} \cdot \mathbf{R}_{q_{x}}}\left(x_{l \alpha}\right) \mathbf{s}_{\mathbf{q}},
$$

where $N_{y} N_{z}$ is the total number of atoms in a cross section, $u_{q_{x}}\left(x_{l \alpha}\right)$ is a one-dimensional phonon wave function, $\mathbf{s}_{\mathbf{q}}$ is the unit vector in the direction of atomic vibrations, and

$$
\mathbf{R}=\left(\begin{array}{c}
y_{l \alpha} \\
z_{l \alpha}
\end{array}\right), \quad \mathbf{Q}=\left(\begin{array}{c}
q_{y} \\
q_{z}
\end{array}\right)
$$

The orthonormalization conditions, Eq. (2), are now rewritten for $u_{q_{x}}(x)$ as

$$
\sum_{\mathbf{n}} u_{q_{x}}^{*}\left(x_{n}\right) u_{q_{x}^{\prime}}\left(x_{n}\right)=\delta_{q_{x} q^{\prime}}
$$

where $x_{n}\left(1,2, \ldots, N_{x}\right)$ is the $x$ component of the $n_{\text {th }}$ lattice point coordinate. For conciseness, we introduce

$$
\left\{\begin{array}{l}
S_{q_{x}}(x) \equiv \frac{1}{\sqrt{M_{\alpha}}} u_{q_{x}}(x) \\
C_{\mathbf{q}} \equiv \sqrt{\frac{\hbar}{2 \omega_{\mathbf{q}}} \frac{1}{N_{y} N_{z}}},
\end{array}\right.
$$

yielding

$$
\mathbf{S}(\mathbf{r})=\sum_{\mathbf{q}} C_{\mathbf{q}}\left(a_{\mathbf{q}}+a_{-\mathbf{q}}^{+}\right) e^{i \mathbf{Q} \cdot \mathbf{R}} S_{q_{x}}(x) \mathbf{s}_{\mathbf{q}},
$$

where the function $S_{q_{x}}(x)$ is the one-dimensional (1D) phonon normal mode.

In this work the function $S_{q_{x}}(x)$ is calculated numerically using the linear atomic chain model shown in Fig. 2. In this model the 1DSiQDA consists of $\mathrm{Si}$ region containing only $\mathrm{Si}$ atoms and the oxide region containing $\mathrm{Si}$ and $\mathrm{O}$ atoms alternating with each other. The lattice potential energy is expanded as a power series of the displacement of the atoms from their minimum-energy positions, but only to the second order, which leads to atomic force proportional to the displacement. The proportionality coefficient acts as "spring constant" of a bond. For simplicity, we consider interactions 


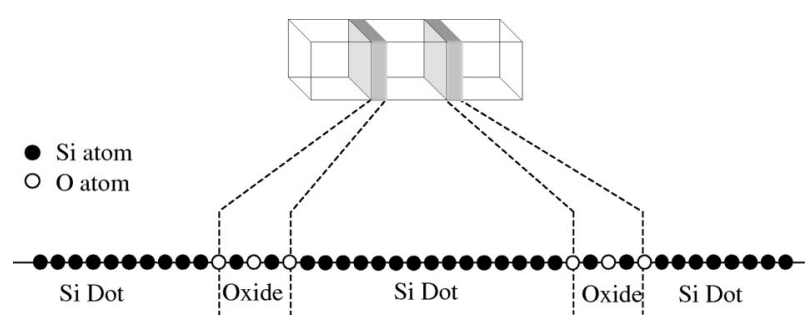

FIG. 2. Illustration of the linear atomic chain model used to obtain numerically one-dimensional phonon normal modes in the 1DSiQDA.

between the nearest atoms only. The phonon normal modes are obtained by solving equations of motion for these atoms under the periodic boundary condition. ${ }^{16}$

Table II shows the parameters used in our simulation. The distance between the neighboring atoms, $a$, is set as $0.25 \mathrm{~nm}$, determined from the mass density of atoms in $\mathrm{Si}$. We use the same distance for $\mathrm{Si}-\mathrm{O}$ bonds. The number of atoms included in a $\mathrm{Si}$ or $\mathrm{SiO}_{2}$ region is obtained by dividing the size by $a$, and the $\mathrm{Si} / \mathrm{SiO}_{2}$ interfaces are set at the $\mathrm{O}$ atoms. The mass of $\mathrm{Si}$ and $\mathrm{O}$ atoms was set based on their atomic weights, that is, $M_{\mathrm{SI}}=16.0 / N_{A} \quad[\mathrm{~g}]$ and $M_{\mathrm{O}}$ $=28.09 / N_{A}[\mathrm{~g}]$ where $N_{A}$ is the Avogadro constant. The spring constant between each $\mathrm{Si}-\mathrm{Si}$ bond, $K_{\mathrm{Si}-\mathrm{Si}}$, is determined so that the phonon maximum energy obtained by the calculation for the SiQW is identical to the maximum energy of longitudinal phonons in bulk Si (100) direction. The proportionality constant between each $\mathrm{Si}-\mathrm{O}$ bond, $K_{\mathrm{Si}-\mathrm{O}}$, is determined from the ratio of Young's modulus for bulk $\mathrm{Si}$ and $\mathrm{SiO}_{2}$ film, that is, $K_{\mathrm{Si}-\mathrm{Si}}: K_{\mathrm{Si}-\mathrm{O}}=180 \mathrm{GPa}: 70 \mathrm{GPa}{ }^{14,15}$ The acoustic deformation potential $D_{\text {aco }}$ in the $\mathrm{Si}$ and oxide region is set based on the published values. ${ }^{12,20}$

\section{Electron-phonon interaction}

The probability of the transition from an initial electronic/phononic state $(\mathbf{k}, c)$ to a final state $\left(\mathbf{k}^{\prime}, c^{\prime}\right)$ is calculated using Fermi's golden rule, and the perturbation Hamiltonian for the ADP scattering can be written, using the phonon wave function $\mathbf{S}(\mathbf{r})$, as ${ }^{12,13}$

$$
H_{\mathrm{ADP}}(\mathbf{r})=D_{\text {aco }} \nabla \mathbf{S}(\mathbf{r}),
$$

where $D_{\text {aco }}$ is the acoustic deformation potential. Using Eq. (8), one finds that the matrix element in the transition probability reads

TABLE II. Parameters used in the simulation.

\begin{tabular}{ccc}
\hline \hline Parameter & Symbol (unit) & Value \\
\hline Atom spacing & $a(\mathrm{~nm})$ & 0.25 \\
Atomic mass of $\mathrm{Si}$ & $M_{\mathrm{Si}}(\mathrm{kg})$ & $46.622 \times 10^{-27}$ \\
Atomic mass of $\mathrm{O}$ & $M_{\mathrm{O}}(\mathrm{kg})$ & $26.559 \times 10^{-27}$ \\
Spring constant $\mathrm{Si}-\mathrm{Si}$ & $K_{\mathrm{Si}-\mathrm{Si}}(\mathrm{N} / \mathrm{m})$ & 103.4 \\
Spring constant $\mathrm{Si}-\mathrm{O}$ & $K_{\mathrm{Si}-\mathrm{O}}(\mathrm{N} / \mathrm{m})$ & 41.36 \\
Acoustic deformation & $D_{\mathrm{aco}}(\mathrm{eV})$ & 9.0 \\
potential in $\mathrm{Si}$ & $D_{\mathrm{aco}}(\mathrm{eV})$ & 3.5 \\
Acoustic deformation & & \\
potential in $\mathrm{SiO}$ & & \\
\hline \hline
\end{tabular}

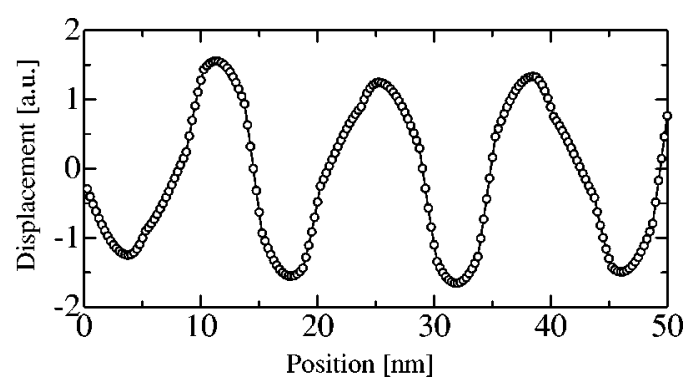

FIG. 3. The real part of a phonon normal mode $\operatorname{Re}\left[S_{q_{x}}(x)\right]$, that is, atomic displacement as a function of position $x$, having a phonon energy of $2.9 \mathrm{meV}$. The Si dot size is set at $4.0 \mathrm{~nm}$, and the oxide thickness is $1.0 \mathrm{~nm}$

$$
\begin{aligned}
\left\langle\mathbf{k}^{\prime} c^{\prime}\left|H_{\mathrm{ADP}}(\mathbf{r})\right| \mathbf{k} c\right\rangle= & D_{\mathrm{aco}}\left(\begin{array}{c}
C_{\mathbf{q}} \sqrt{n_{\mathbf{q}}} \\
C_{-\mathbf{q}} \sqrt{n_{-\mathbf{q}}+1}
\end{array}\right)\left\langle k_{y}^{\prime} k_{z}^{\prime}\left|e^{ \pm i \mathbf{Q} \cdot \mathbf{R}}\right| k_{y} k_{z}\right\rangle \\
& \times\left\{ \pm i\left(q_{y} s_{ \pm q, y}+q_{z} s_{ \pm q, z}\right)\left\langle k_{x}^{\prime}\left|S_{ \pm q_{x}}(x)\right| k_{x}\right\rangle\right. \\
& \left.+s_{ \pm q, x}\left\langle k_{x}^{\prime}\left|\frac{\partial S_{ \pm q_{x}}(x)}{\partial x}\right| k_{x}\right\rangle\right\} .
\end{aligned}
$$

It is important to note that the integral $\left\langle k_{y}^{\prime} k_{z}^{\prime}\left|e^{ \pm i \mathbf{Q} \cdot \mathbf{R}}\right| \mid k_{y} k_{z}\right\rangle$ can be regarded as the Fourier transform of a function localized within $0<y<L_{y}$ and $0<z<L_{z}$. Therefore, the integral is nonzero only when the $y$ and $z$ components of the phonon wave vector satisfy $q_{y} \sim 1 / L_{y}$ and $q_{z} \sim 1 / L_{z}$. For $L_{y}=L_{z}$ $=4 \mathrm{~nm}$, which is a typical Si dot size in the literature, ${ }^{2-4,21} \mathrm{a}$ phonon vector satisfying the above criteria has an energy $\sim 1 \mathrm{meV}$ in the $y$ and $z$ directions. As the $x$ component of the phonon energy can range up to about $60 \mathrm{meV},{ }^{22}$ majority of phonons contributing to the electron-phonon scattering have a wave vector parallel to the $x$ axis. Therefore, the following approximation is valid:

$$
\left|\left\langle\mathbf{k}^{\prime} c^{\prime}\left|H_{\mathrm{ADP}}\right| \mathbf{k} c\right\rangle\right|^{2} \propto\left|\left\langle k_{x}^{\prime}\left|D_{\mathrm{aco}} \frac{\partial S_{ \pm q_{x}}(x)}{\partial x}\right| k_{x}\right\rangle\right|^{2} .
$$

Thus, in the 1DSiQDA, the one-dimensional phonon normal mode $S_{ \pm q_{x}}(x)$ plays the major role in the acoustic deformation potential scattering. In the following sections, we analyze behaviors of $S_{ \pm q_{x}}(x)$ and the one-dimensional ADP scattering potential in the right-hand side of the Eq. (11).

\section{RESULTS}

\section{A. Phonon normal modes and ADP scattering potential}

Figure 3 shows the atomic displacement at each equilibrium point, which is given by the real part of a phonon normal mode as a function of position $x, \operatorname{Re}\left[S_{q_{x}}(x)\right]$. The phonon energy is set as $2.9 \mathrm{meV}$. Each open circle represents an atom, and the interpolating solid line is merely for visualization. In the calculation, the $\mathrm{Si}$ dot size is set as $4.0 \mathrm{~nm}$, and the oxide thickness is $1.0 \mathrm{~nm}$, so that the regions $5.0 \mathrm{n}-1.0$ $<x<5.0 n,(n=1,2, \ldots)$ correspond to oxide layers. The wave form apparently differs from a plane wave expected in the SiQW and is distorted at the oxide layers. Note that, in the oxide layers, displacement differences between neighbor- 


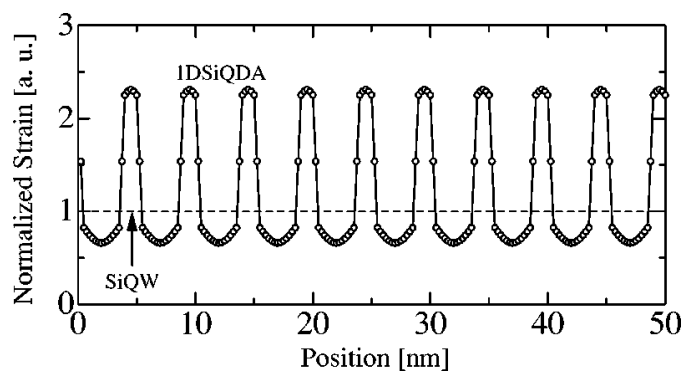

FIG. 4. The absolute value of the first derivative of the normal mode, $|\varepsilon(x)|$, calculated from the result shown in Fig. 3, which is associated with strain caused by the phonon vibration. The broken line is the result obtained from a phonon normal mode in the silicon quantum wire (SiQW) at the same phonon energy. The vertical axis is normalized so that the result for the SiQW becomes unity.

ing atoms are larger than that in the Si dot region. This is due to the fact that the atoms have larger separations due to the smaller spring constant in the oxides.

Figure 4 shows the absolute value of the first derivative of the normal mode in terms of position, $\varepsilon(x)=\partial S_{q_{x}}(x) / \partial x$, calculated from the result shown in Fig. 3 using the following formula:

$$
\left|\varepsilon\left(x_{n}\right)\right|=\left|\frac{S_{q_{x}}\left(x_{n+1}\right)-S_{q_{x}}\left(x_{n-1}\right)}{2 a}\right| .
$$

This quantity appears on the right-hand side of Eq. (11) and is associated with the strain caused by the phonon vibration. The broken line in the figure is the result obtained from a phonon normal mode in the SiQW having the same energy, which is obtained by taking all atoms as Si. The vertical axis is normalized so that the results for the SiQW become unity. Note that the strain is larger in the oxide layers than in the $\mathrm{Si}$ dots. This is, of course, due to the large distortion in the oxide layers observed in Fig. 3. Also note that the strain in the $\mathrm{Si}$ dots is weaker compared to that in the SiQW. This indicates that the strain in the $\mathrm{Si}$ dots is effectively "absorbed" by the oxide layers inserted between these dots. This is reasonable because oxide layers are softer than $\mathrm{Si}$ dots. In this paper, this effect is referred to as "strain absorption effect."

Figure 5 shows the absolute value of the onedimensional ADP scattering potential as a function of position. It is obtained from the result shown in Fig. 4 using the following expression:

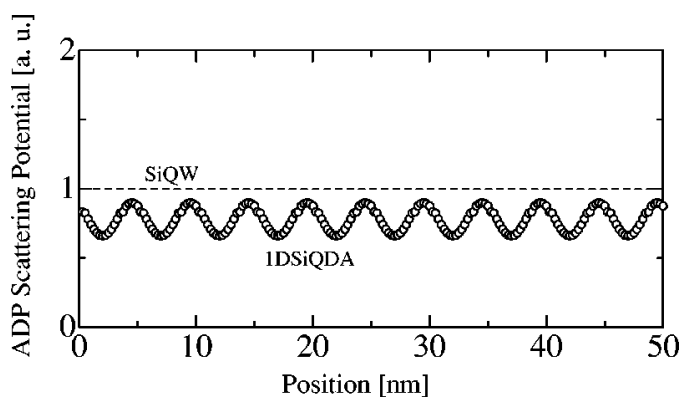

FIG. 5. The absolute value of the acoustic deformation potential (ADP) scattering potential as a function of position. The broken line is the result for a phonon in the SiQW having the same energy.

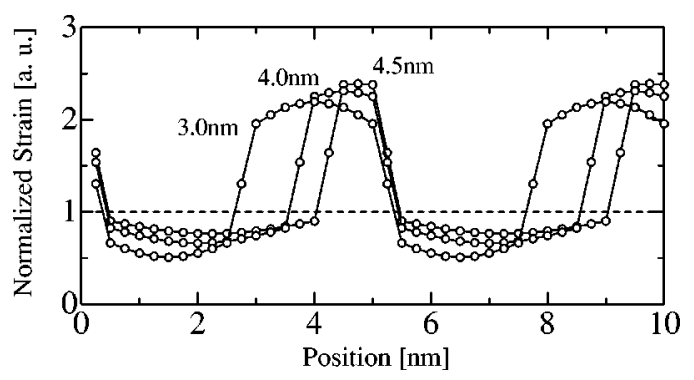

FIG. 6. The strain profile $|\varepsilon(x)|$ plotted for $L_{x}=3.0,4.0$, and $4.5 \mathrm{~nm}$. The broken line is the result obtained for the SiQW. All the phonon modes are at $2.9 \mathrm{meV}$.

$$
\left|H_{\mathrm{ADP}}\left(x_{n}\right)\right|=D_{\mathrm{aco}}\left(x_{n}\right)\left|\varepsilon\left(x_{n}\right)\right| \text {. }
$$

It is important to note that the ADP scattering potential is weaker than that in the SiQW at any position. The above observation is true even at the oxide layers, where there is considerably larger strain. This is due to the fact that the acoustic deformation potential $D_{\text {aco }}(x)$ is smaller in the oxide layers than in the Si. In other words, the oxide layers having relatively weak electron-acoustic-phonon coupling absorbed the strain from Si dots having strong coupling, resulting in the reduction of the ADP scattering potential all over the 1DSiQDA.

\section{B. Si dot size dependence of ADP reduction}

For device application, it is important to investigate the impact of Si dot size variation on the ADP scattering potential reduction. As shown above, the scattering potential reduces due to the strain absorption of oxide layers, so that the ratio of $\mathrm{Si}$ dot size to the oxide layer thickness $L_{x} / T_{\mathrm{ox}}$ is an essential parameter. For this reason, in the following analysis, the Si dot size is changed so that the sum $L_{x}+T_{\text {ox }}$ remains $5.0 \mathrm{~nm}$.

Figure 6 shows the absolute value of the first derivative of the normal mode plotted for $L_{x}=3.0,4.0$, and $4.5 \mathrm{~nm}$. For each Si dot size, phonon modes having the energy of $2.9 \mathrm{meV}$ are chosen. Note that the strain in the Si dots decreases as $L_{x}$ decreases. This indicates that the 1DSiQDA with thicker oxide layer leads to less ADP scattering potential. We have confirmed that this is true for phonon normal modes at different energies.

Figure 7 shows the average ADP scattering potential as a function of phonon energy. The average ADP scattering potential is obtained by averaging $\left|H_{\mathrm{ADP}}(x)\right|$ over the 1DSiQDA:

$$
\left\langle\left|H_{\mathrm{ADP}}\right|\right\rangle=\frac{1}{N_{x}} \sum_{n=1}^{N_{x}}\left|H_{\mathrm{ADP}}\left(x_{n}\right)\right|,
$$

where $N_{x}$ denotes the total number of atoms included in a period. In the figure, the results obtained for different $L_{x}$ are plotted with solid curves, and those for the SiQW and oxide wire $\left(L_{x}=0\right)$ are plotted with broken curves. Note that the scattering potential decreases monotonically with decreasing Si dot size, and the amount of the reduction is almost the same at any phonon energy. This indicates that the reduction 


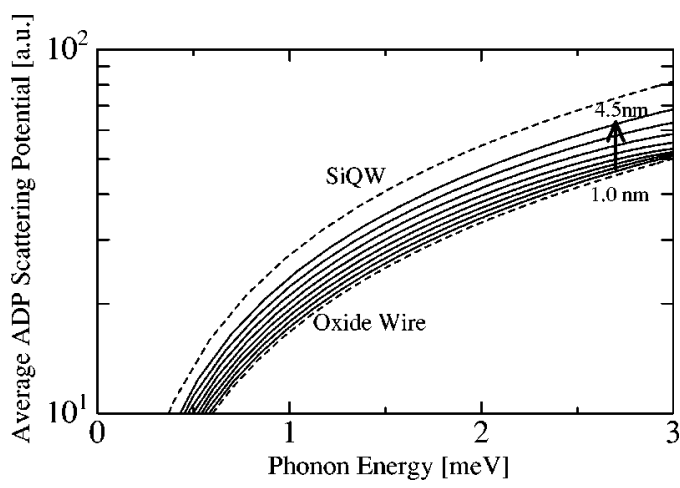

FIG. 7. The average ADP scattering potential as a function of phonon energy plotted for $L_{x}=1.0,1.5,2.0,2.5,3.0,3.5,4.0$, and $4.5 \mathrm{~nm}$. The results for the SiQW and oxide wire are also shown with broken lines.

of the scattering potential is universal to any phonon modes and depends solely on structural parameters.

Figure 8 shows the average scattering potential as a function of $\mathrm{Si}$ dot size $L_{x}$ (and therefore, oxide thickness $\left.T_{\mathrm{ox}}\right)$. The data are obtained from Fig. 7 at a phonon energy of $2.9 \mathrm{meV}$. The average scattering potential is reduced up to $40 \%$, depending on the $\mathrm{Si}$ dot size. Note that the average scattering potential does not vary linearly with the Si dot size. This supports the idea that the strain absorption effect plays an important role in the reduction of scattering potential, because otherwise the scattering potential would decrease merely due to the increase of oxide layer volume, where $D_{\text {aco }}$ is small. At small dot size, decreasing Si dot size does not dramatically contribute to the reduction of the scattering potential. This indicates that the reasonable $L_{x} / T_{\text {ox }}$ ratio for exploiting the reduction of scattering potential would be around 1.5 or higher.

\section{DISCUSSION}

In this section, we discuss several issues regarding the results obtained in the previous section.

First, we discuss how the reduction of the ADP scattering potential affects the phonon scattering rate. It is obvious that the reduction of the scattering potential leads to the reduction of the scattering rate. However, one should notice

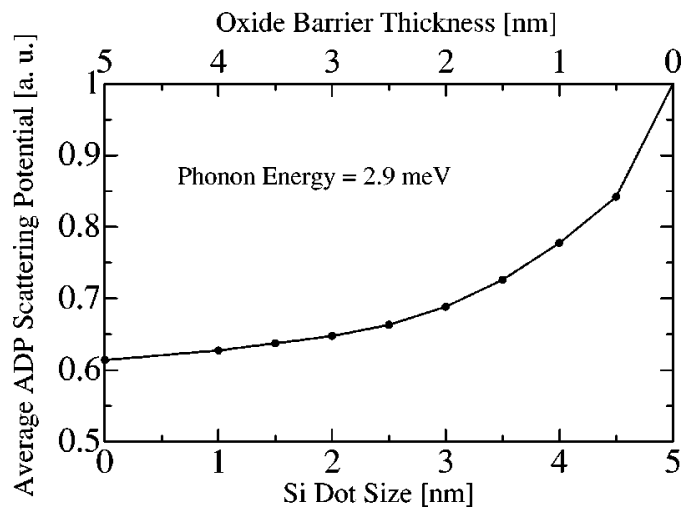

FIG. 8. The average ADP scattering potential as a function of Si dot size $L_{x}$ The $\mathrm{Si}$ dot size is changed so that the sum $L_{x}+T_{\text {ox }}$ remains at $5.0 \mathrm{~nm}$. The data are extracted from Fig. 7 at a phonon energy of $2.9 \mathrm{meV}$ and normalized to the value for the SiQW. that introducing the oxide layers between the $\mathrm{Si}$ dots also alters the electronic states. For example, when the electric field applied along the $x$ axis of the 1DSiQDA is negligible, the potential seen by electrons is the periodic Krönig-Penny potential, resulting in the miniband structure. This change in the electronic state also affects the scattering rate, because it includes electron wave functions. For this reason, it is difficult to conclude straightforwardly that the reduction of the ADP scattering potential results in the reduction of scattering rate. Although the calculation of the scattering rate is outside the scope of this paper, its evaluation is required to predict electron transport in the 1DSiQDA. Provided the scattering rate is reduced, this reduction would not increase the mobility along the $x$ axis, because inserted oxide layers apparently impede the transport of electrons. However, the reduced scattering rate may lead to a longer coherent time in this system, because the ADP scattering is the major mechanism of decoherence on the electronic states in this system. This may be advantageous for devices utilizing coherent quantum states.

The second issue is the polar-optical phonon scattering from $\mathrm{Si}-\mathrm{O}$ dipoles in the oxide layer. Silicon oxide is a polar material, and POP scattering is the major scattering mechanism for low-energy electrons. ${ }^{23}$ This scattering mechanism would be negligible as long as the ratio $L_{x} / T_{\text {ox }}$ is large enough. However, when this ratio is small, the POP scattering may not be negligible even in the 1DSiQDA. In order to take advantage of the reduction of the ADP scattering potential, the ratio $L_{x} / T_{\mathrm{ox}}$ should therefore be large enough to diminish the POP scattering. Considering that the result in Fig. 8 shows sharp decrease at large Si dot size, one would be able to design large reduction in the ADP scattering potential while avoiding POP scattering.

Finally, we comment on applicability of the results obtained here to the experimental results. The essence of the reduction of the ADP scattering potential is to generate strain redistribution in Si nanostructures by mixing them with soft oxide layers. The perfect periodicity and one dimensionality of the 1DSiQDA might not be actually needed for this strain redistribution. Therefore, the conclusions in this paper would also be applicable to the systems mentioned in the Introduction. ${ }^{2,4,6,7}$ In addition, the similar reduction of the ADP scattering potential might emerge in other structures, such as the $\mathrm{SiQW}$ embedded in $\mathrm{SiO}_{2}$ and a silicon slab sandwiched by $\mathrm{SiO}_{2}$. As electrons travel only within $\mathrm{Si}$ region in these cases, the reduction of the ADP scattering potential might lead to significant mobility enhancement. In fact, the possibility of reduced ADP scattering in a slab sandwiched by softer material is implied in Ref. 24. Alteration of the phonon despersion relations in various nanostructures have also been examined, and many interesting phenomena in electron transport and the lattice thermal conductivity have been predicted. ${ }^{25,26}$ Such phonon engineering in $\mathrm{Si}$ and $\mathrm{SiO}_{2}$ systems could be one of the most important topics in future Si-based nanoelectronics.

\section{CONCLUSIONS}

We have investigated the ADP scattering in the 1DSiQDA. An approximate expression for the acoustic- 
phonon waves in the 1DSiQDA has been derived, and it has been shown that the one-dimensional phonon normal modes along the 1DSiQDA play major role in the ADP scattering. The 1D phonon normal modes were numerically obtained using the linear atomic chain model. The simulation results have revealed that the strain caused by the phonon vibration is absorbed by the oxide layers, resulting in reduction of the strain in the Si dots. This strain absorption effect eventually leads to the reduction of the ADP scattering potential all over the $1 \mathrm{DSiQDA}$. The average scattering potential is reduced up to $40 \%$ compared to that in the SiQW, depending on the $\mathrm{Si}$ dot size. The amount of the reduction does not depend on the phonon energy, but rather on the ratio $L_{x} / T_{\text {ox }}$. The above results indicate great potential for phonon engineering by mixing $\mathrm{Si}$ and silicon oxide layers.

\section{ACKNOWLEDGMENTS}

The authors are indebted to Dr. D. Williams of Hitachi Cambridge Laboratory and Professor S. Oda of Tokyo Institute of Technology for their support.

${ }^{1}$ N. Koshida, T. Ozaki, X. Sheng, and H. Koyama, Jpn. J. Appl. Phys., Part 2 34, L705 (1995)

${ }^{2}$ N. Koshida, X. Sheng, and T. Komoda, Appl. Surf. Sci. 146, 371 (1999).

${ }^{3}$ T. Komoda, X. Sheng, and N. Koshida, J. Vac. Sci. Technol. B 17, 1076 (1999).

${ }^{4}$ K. Nishiguchi, X. Zhao, and S. Oda, J. Appl. Phys. 92, 2748 (2002).

${ }^{5}$ S. Uno, K. Nakazato, S. Yamaguchi, A. Kojima, N. Koshida, and H.
Mizuta, IEEE Trans. Nanotechnol. 2, 301 (2003).

${ }^{6}$ M. Khalafalla, Z. Durrani, and H. Mizuta, Appl. Phys. Lett. 85, 2262 (2004).

${ }^{7}$ Y. Tsuchiya, K. Takai, N. Momo, S. Oda, S. Yamaguchi, T. Shimada, and H. Mizuta, 27th International Conference on Physics of Semiconductors (ICPS-27), Arizona, USA, 2004 (unpublished).

${ }^{8}$ N. Koshida and N. Matsumoto, Mater. Sci. Eng., R. 40, 169 (2002).

${ }^{9}$ T. Ichihara, T. Baba, T. Komoda, and N. Koshida, J. Vac. Sci. Technol. B 24, 1372 (2004).

${ }^{10}$ T. Ichihara, T. Hatai, K. Aizawa, and T. Komoda, J. Vac. Sci. Technol. B 22, 57 (2004).

${ }^{11}$ H. Noguchi, J. P. Leburton, and H. Sakaki, Phys. Rev. B 47, 15593 (1993).

${ }^{12}$ C. Jacoboni and L. Reggiani, Rev. Mod. Phys. 55, 645 (1983).

${ }^{13} \mathrm{M}$. Lundstrom, Fundamentals of Carrier Transport (Cambridge University Press, Cambridge, 2000), p. 60.

${ }^{14}$ B. Bhushan and X. Li, J. Mater. Res. 12, 54 (1992).

${ }^{15}$ M. T. Kim, Thin Solid Films 283, 12 (1996).

${ }^{16} \mathrm{O}$. Madelung, Introduction to Solid-State Theory (Springer, New York, 1996), p. 130.

${ }^{17}$ R. W. Morse, J. Acoust. Soc. Am. 22, 219 (1950).

${ }^{18}$ G. Fishman, Phys. Rev. B 36, 7448 (1987).

${ }^{19}$ T. Yamada and J. Sone, Phys. Rev. B 40, 6265 (1989).

${ }^{20}$ M. V. Fischetti, Phys. Rev. Lett. 53, 1755 (1984).

${ }^{21}$ H. Tanino, A. Kuprin, H. Deai, and N. Koshida, Phys. Rev. B 53, 1937 (1996).

${ }^{22}$ S. M. Sze, Physics of Semiconductor Devices (Wiley, New York, 1981).

${ }^{23}$ M. V. Fischetti, D. J. DiMaria, S. d. Brorson, T. N. Theis, and J. R. Kirtley, Phys. Rev. B 31, 8124 (1985).

${ }^{24}$ E. P. Pokatilov, D. L. Nika, and A. A. Balandin, Appl. Phys. Lett. 85, 825 (2004).

${ }^{25}$ O. L. Lazarenkova and A. A. Balandin, Phys. Rev. B 66, 245319 (2002).

${ }^{26}$ J. Zou and A. Balandin, J. Appl. Phys. 89, 2932 (2001). 